\title{
Carbamazepine restores neuronal signaling, protein synthesis and cognitive function in a mouse model of fragile $X$ syndrome
}

Running title: Carbamazepine corrects symptoms associated with FXS

\author{
Qi Ding ${ }^{1}$, Fan Zhang ${ }^{1 \$}$, Yue Feng ${ }^{3}$, Hongbing Wang ${ }^{1,2, *}$ \\ ${ }^{1}$ Department of Physiology, ${ }^{2}$ Neuroscience Program, Michigan State University, East \\ Lansing, MI 48824; ${ }^{3}$ Department of Pharmacology and Chemical Biology, Emory \\ University School of Medicine, Atlanta, GA 30322.
}

$\$$ current address: College of Life Science and Agriculture, Zhoukou Normal College, Zhoukou, Henan 466000, China

"Address correspondence to Hongbing Wang, Department of Physiology, Neuroscience Program, Michigan State University, East Lansing, MI 48824; Telephone: 517-884-5119; Fax: 517-432-1967; E-mail: wangho@msu.edu. 


\section{ABSTRACT}

Fragile $X$ syndrome (FXS) is a leading genetic disorder of intellectual disability caused by the loss of the functional fragile $X$ mental retardation protein (FMRP). To date, there is no efficacious mechanism-based medication for FXS. With regard to potential disease mechanisms in FXS, it is widely accepted that the lack of FMRP causes elevated protein synthesis and deregulation of neuronal signaling. Abnormal enhancement of the ERK $1 / 2$ (extracellular signal-regulated kinase $1 / 2$ ) and PI3K-Akt (Phosphoinositide 3 kinase-protein kinase B) signaling pathways has been identified in both FXS patients and FXS mouse models. In this study, we show that carbamazepine, which is an FDA-approved drug and has been mainly used to treat seizure and neuropathic pain, corrects cognitive deficits including passive avoidance and object location memory in FXS mice. Carbamazepine also rescues hyper locomotion and social deficits. At the cellular level, carbamazepine dampens the elevated level of ERK $1 / 2$ and Akt signaling as well as protein synthesis in FXS mouse neurons. Together, these results advocate repurposing carbamazepine for FXS treatment. 


\section{INTRODUCTION}

Fragile $X$ syndrome (FXS) is a leading cause of inherited intellectual disability and autism. Genetic studies have demonstrated that loss of function mutations in the fragile $\mathrm{X}$ mental retardation 1 gene (FMR1) that encodes the fragile $\mathrm{X}$ mental retardation protein (FMRP) results in FXS ${ }^{1,2}$. Although the disease mechanism remains largely elusive, multiple lines of data demonstrate that FMRP interacts with both mRNAs and protein translation machinery ${ }^{1-3}$. It has been found that, except for few specific targets ${ }^{4}$, FMRP deficiency causes an overall enhancement of protein synthesis ${ }^{3}$. There are also lines of clinical and preclinical evidence suggesting altered neuronal signaling in FXS. Previous studies have found that activity of the ERK $1 / 2$ signaling pathway is abnormally enhances in FXS mouse model (i.e. Fmr1 knockout mouse) and human patient samples ${ }^{5-7}$. With regards to understanding how alteration of FMRP-regulated translation is linked to elevated ERK1/2 activity, it is found that FMRP binds Adcy1 (type 1 adenylyl cyclase) mRNA $^{8,9}$, and ADCY1 protein level is elevated in Fmr1 knockout (KO) mice ${ }^{10}$. Consistent with the finding that enhancement of ADCY1 expression causes elevated ERK1/2 activity $^{11}$ and a panel of FXS-associated behavior (e.g. hyperlocomotion and social deficits) ${ }^{12}$, genetic reduction of Adcy1 attenuates the elevated ERK1/2 signaling and the aforementioned behavioral abnormalities in Fmr1 KO mice ${ }^{10}$. Intriguingly, genetic reduction of Adcy1 also restores Akt signaling ${ }^{10}$, the activity of which is elevated in both human patient and FXS mouse samples ${ }^{7,13,14}$. These observations suggest that abrrant hyperfunction of ADCY1 in FXS is a potential therapeutic target, through which the pathological overactivation of both ERK1/2 and Akt signaling pathways can be corrected. However, the potential therapeutic value in clinical application requires examination with practical pharmacological intervention.

Carbamazepine is an FDA-approved drug and is currently used for treatment of seizure and neuropathic pain. Because carbamazepine shows pharmacological activity against $A D C Y 1{ }^{15}$, we reason that it may elicit therapeutic effects on FXS-associated pathological phenotypes. In contrast to ADCY, which contributes to CAMP production, PDE (phosphodiesterase) possesses enzymatic activity to reduce cAMP level by converting it to AMP. Notably, it is reported that FMRP also regulates the translation of certain PDE subtype (e.g. PDE2A) and the PDE2A protein level is elevated in Fmr1 KO mice ${ }^{16}$. Thus, it is intriguing and seemingly possible that FMRP deficiency could concurrently affect signaling molecules with counteracting enzymatic activity.

To better understand this complication and the therapeutic potential through inhibiting ADCY or PDE2, in this study, we compared ADCY1 and PDE2A expression in wild type (WT) and Fmr1 KO mice. Furthermore, we demonstrated that inhibnition of ADCY 1 by carbamazepine can correct abnormal ERK1/2 and Akt signaling, protein synthesis, and the core symptoms of cognitive deficits in a mouse model of FXS. However, PDE2A inhibitors can only attenuate the elevated Akt activity but have no effect on correcting the abnormal ERK1/2 activity and protein synthesis. These results provide the first evidence demonstrating differential pathological contributions of emzymatic components in cAMP homeostasis in FXS.

\section{MATERIALS AND METHODS}

Animals 
WT and Fmr1 KO mice were generated by breeding WT males with Fmr1 heterozygous females. For all behavioral examinations, 2.5- to 3-month old male mice were used. Animals had free access to food and water and were housed in the Campus Animal Research facility under $12 \mathrm{~h}$ light/dark cycle. All procedures used in the study were approved by the Institutional Animal Care and Use Committee at Michigan State University.

Cell culture

Neurons ${ }^{17}$ and glial cells ${ }^{18}$ were cultured from postnatal day 0 WT and Fmr1 KO mice. Briefly, the cortex and hippocampus were harvested and digested to obtain dissociated neurons and glial cells. Neurons were grown in Neurobasal A supplemented with B27, $0.5 \mathrm{mM}$ glutamine and antibiotics and one-third of the growth media was replaced with fresh media once every 3 days. For glia culture, the dissociated cells were maintained in DMEM supplemented with $10 \%$ FBS and antibiotics for 9 days and then one-third of the media was replaced with fresh media once every 3 days.

Western blot analysis

Samples were first harvested from primary cell cultures on DIV (days in vitro) 14 or from brain tissues of adult (2.5- to 3-month old) mice. After sonication, samples were subjected to $4-20 \%$ SDS-PAGE and transferred onto nitrocellulose membranes. The membranes were first incubated with primary antibodies overnight at $4{ }^{\circ} \mathrm{C}$, washed with PBST (PBS with $0.1 \%$ Triton-X100), incubated with IRDye $800 \mathrm{CW}$ goat anti-rabbit or IRDye 680RD goat anti-mouse secondary antibodies (LI-COR Biosciences, Cat\#926-32211 and Cat\#926-68070, respectively, 1:5000) for 2 hours at room temperature, and washed with PBST. The signals were detected by the Odyssey digital imaging system (LI-COR Biosciences). The primary antibodies were rabbit anti-ADCY1 (Sigma, Cat\# SAB4500146, 1:1000), rabbit anti-ERK1/2 (Cell Signaling, Cat\#9102, 1:1000), rabbit antipERK1/2 (Cell Signaling, Cat\#9101, 1:2000), rabbit anti-Akt (Cell Signaling, Cat\#7272, $1: 1000)$, rabbit anti-pAkt (Cell Signaling, Cat\#13038, 1:1000), and mouse anti- $\beta$-actin (Sigma, Cat\#A5541, 1:10,000).

In vivo drug administration

Vehicle (10\% DMSO) or $20 \mathrm{mg} / \mathrm{kg}$ carbamazepine was i.p. injected $1 \mathrm{~h}$ before the behavioral examinations. The examination of passive avoidance memory and object location memory involved training and testing; animals received i.p. injection $1 \mathrm{~h}$ before training and were tested 24 hours later. For repeated drug administration, the animals received daily injection of vehicle or $20 \mathrm{mg} / \mathrm{kg}$ carbamazepine for 8 days, and behavior examinations were performed $1 \mathrm{~h}$ after the last injection.

Behavioral examination

For passive avoidance memory test ${ }^{19}$, mouse was placed in the lit side of a passive avoidance chamber (Coulbourn Instruments, Whitehall, PA) and allowed to explore for 1 min, following which the trap door was opened. Once the animal crossed over to the dark side, the trap door was closed and a mild foot shock $(0.7 \mathrm{~mA}$ for $2 \mathrm{sec})$ was delivered. The mouse remained in the dark side for $30 \mathrm{sec}$ before being returned to its home cage. After $24 \mathrm{~h}$, the trained mouse was reintroduced to the lit side and the crossover latency 
to the dark side was recorded. A maximum of $300 \mathrm{sec}$ was used as the cutoff crossover latency value.

For object location memory test ${ }^{20}$, mouse was first habituated by exposing to the empty training chamber for 10 min daily for 3 consecutive days. On day 4 , the mouse was exposed to the chamber with two objects placed at location A and B. After the 10-min training, the mouse was returned to its home cage. Twenty-four hours later on day 5, the trained mouse was re-introduced to the same chamber with the same objects. One object was placed at the old location $A$ and the other object was place at the new location $C$. The percentage of time that mouse spent with object at location $A$ and $B$ during training or $\mathrm{A}$ and $\mathrm{C}$ during testing was scored. Discrimination index was calculated as [(time spend with location $\mathrm{C}$ - time spent with location $\mathrm{A}$ )/total time spent with objects at both location $A$ and $\mathrm{C}$ ] during testing.

For open field test ${ }^{19}$, mouse was placed in the center of an open field arena and allowed to move freely for $1 \mathrm{~h}$. Ambulatory travel distance in the whole arena and in the center area of the arena as well as the number of entry to the center area were recorded by the TurScan Activity System (Coulbourn Instruments, Whitehall, PA).

For social interaction test, we used a 3-chamber paradigm ${ }^{21}$ that has been sufficient to detect social deficits in FXS mouse model ${ }^{22,23}$. Briefly, mouse was placed in the center of a 3-chamber social interaction box and allowed to freely explore the entire box for 5 min. Then the mouse was briefly restricted in the center compartment and a stimulus mouse was placed in a wire enclosure on one side and an empty wire enclosure on the other side. The time spent in mouse chamber and in sniffing the stimulus mouse was recorded for $10 \mathrm{~min}$.

For light/dark test ${ }^{19}$, mouse was placed in the dark side of a light/dark chamber and habituated for $2 \mathrm{~min}$, after which the trap door was opened. The mouse was allowed to move freely between two chambers. The time spent in the lit chamber and the number of crossing to the lit chamber were recorded for $5 \mathrm{~min}$.

Drug effects on protein synthesis and neuronal signaling Protein synthesis was determined by the SUnSET method, which involves labelling with puromycin ${ }^{24}$. To determine the drug effect, DIV 14 hippocampal neurons were pre-treated with carbamazepine $(10,40,100 \mu \mathrm{M})$, BAY607550 $(0.2,1,5 \mu \mathrm{M})$, or forskolin (1 and 5 $\mu \mathrm{M})$ for $30 \mathrm{~min}$ followed by $30 \mathrm{~min}$ incubation with $5 \mu \mathrm{g} / \mathrm{ml}$ puromycin. Samples were harvested at the end of treatment, and protein synthesis level was determined by Western blot using anti-puromycin antibody (KeraFAST, Cat\#EQ0001, 1:1000). The combined intensity of proteins ranging from 20 to $200 \mathrm{kDa}$ was quantified and normalized to the level of $\beta$-actin.

To determine the drug effects on neuronal signaling, DIV 14 hippocampal neurons were treated with various concentrations of carbamazepine, BAY607550, or forskolin for $1 \mathrm{~h}$. The treated neurons were harvested. The level of ERK1/2, pERK1/2, Akt, pAkt, and $\beta$-actin was determined by Western Blot.

Statistical analysis

Student's t-test was used to analyze data between two groups. Two-way ANOVA followed by Holm-Sidak test was used to analyze the genotype effect and treatment effect. Threeway ANOVA followed by Holm-Sidak test was used to analyze the genotype effect, 
treatment effect, and location effect for data collected from object location memory test. Three-way repeated measures ANOVA was used to analyze the open field data at various time points. Since the crossover latency during testing in passive avoidance is not normally distributed, the numerical values were converted to categorical data (cut-off value is $300 \mathrm{sec}$ ) and then analyzed by Fisher's exact test. All data are presented as average \pm SEM.

\section{RESULTS}

ADCY1 is expressed in neurons and elevated in the dorsal hippocampus of Frm1 KO mice

Adcy 1 is predominantly expressed in the central nervous system (CNS) ${ }^{25,26}$, making it an attractive drug target for the treatment of neurological disorders. Here, we further found that ADCY1 is expressed in neurons but not glial cells. The enrichment of neurons or glial cells in our primary cultures was confirmed by immunoblots using antibodies against neuronal and glial-specific marker proteins (Fig.1). Clearly, ADCY1 expression is restricted in neurons enriched of synapsin I (Fig. 1a). Extracts from glial culture show expression of glial fibrillary acidic protein (GFAP) but not ADCY1 (Fig. 1a). Interestingly, while ERK1/2 show robust expression in both cell types, Akt is predominately expressed in neurons, far exceeding that in glial cells (Fig. 1a).

FMRP interacts with Adcy1 mRNA and suppress its translation ${ }^{8-10}$. We confirmed that ADCY1 protein level is elevated in the hippocampus of Fmr1 KO mice (Fig. 1b). Interestingly, the elevated ADCY1 expression is brain region specific. It is prefernetilaly elevated in the dorsal hippocampus but not in ventral hippocampus and prefrontal cortex of Fmr1 KO mice (Fig. 1c).

Carbamazepine restores hippocampus-dependent memory in Frm1 KO mice

Recapitulating the intellectual disability in human FXS patients, Fmr1 KO mice display deficits in various learning and memory tasks including the hippocampus-dependent passive avoidance memory ${ }^{19,27}$. We administered carbamazepine through i.p. injection followed by passive avoidance training. The carbamazepine- and vehicle-treated WT and Fmr1 KO mice showed comparable crossover latency during training (genotype effect: $F_{1,39}=3.404, p=0.073$; treatment effect: $F_{1,39}=1.459, p=0.234$; genotype $X$ treatment interaction: $F_{1,39}=1.501, p=0.228$ ) (Fig. 2a). When tested 24 hours later, the vehicletreated Fmr1 KO mice showed significantly shorter crossover latency than the vehicletreated WT mice ( $p=0.009$, Fisher exact test). Carbamazepine improved the memory in Fmr1 KO mice to the WT level ( $p=0.495$, Fisher exact test). (Fig. 2a).

We next tested the effects of carbamazepine on object location memory (Fig. 2b1), which mainly depends on dorsal hippocampus ${ }^{28,29}$. We found that Fmr1 KO mice are impaired to establish object location memory. While all groups of animals showed comparable preference for objects in location $A$ and $B$ during training (Fig. 2b2) (genotype effect: $F_{1,62}=0.000, p=1.000$; treatment effect: $F_{1,62}=0.000, p=1.000$; genotype $X$ treatment interaction: $\left.F_{1,62}=3.691, p=0.059\right)$, the vehicle-treated WT but not $F m r 1 \mathrm{KO}$ mice showed preference for the object in the new location (i.e. location $\mathrm{C}$ ) over the object in the old location (i.e. location A) during testing (Fig. 2b3) (WT: $p=0.018$; Fmr1 KO: $p=0.991)$. The carbamazepine-treated WT and Fmr1 KO mice both showed preference for the new location (Fig. 2b3) (genotype $X$ location: $F_{1,62}=6.240, p=0.015$; treatment $X$ 
location: $F_{1,62}=11.310, p=0.001$; genotype $X$ treatment $X$ location: $F_{1,62}=0.003, p=0.955$; WT: $p=0.000$; Fmr1 KO: $p=0.001$ ). However, a single injection of carbamazepine did not improve object discrimination index in Fmr1 KO mice (Fig. 2b4) (genotype effect: $F_{1}$, ${ }_{31}=3.12, p=0.087$; treatment effect: $F_{1,31}=5.655, p=0.024$; genotype $X$ treatment interaction: $\left.F_{1,31}=0.002, p=0.968\right)$. Interestingly, $F m r 1 \mathrm{KO}$ mice receiving repeated daily administration of carbamazepine for 8 days displayed normal behavior during training (Fig. 2c1) (genotype effect: $F_{1,62}=0.000, p=1.000$; treatment effect: $F_{1,62}=0.000, p=1.000$; genotype $X$ treatment interaction: $\left.F_{1,62}=0.003, p=0.959\right)$. They showed improved object location memory (Fig. 2c2) (vehicle: $p=0.299$; carbamazepine: $p=0.000$ ) and object discrimination index during testing (Fig. $2 c 3$ ) (genotype effect: $F_{1,31}=0.396, p=0.534$; treatment effect: $F_{1,31}=0.846, p=0.365$; genotype $X$ treatment interaction: $F_{1,31}=9.819$, $p=0.004)$.

Effects of carbamazepine on non-cognitive behavioral abnormalities in Frm1 KO mice Besides intellectual disability, other core FXS behavioral symptoms include hyperactivity and social deficits. We examined the effects of carbamazepine on locomotion activity. One hour after carbamazepine or vehicle injection, mice were placed in an open field arena. Consistent with previous studies ${ }^{2,19}$, vehicle-treated $F m r 1 \mathrm{KO}$ mice showed hyperlocomotion than WT mice in the whole arena (Fig. 3a1 and 3a1'; genotype effect: $F_{1}$, ${ }_{52}=23.814, p=0.000$; treatment effect: $F_{1,52}=0.222, p=0.640$; genotype $X$ treatment interaction: $F_{1,52}=6.147, p=0.016$ ) and the center area of the arena (Fig. 3a2 and 3a2'; genotype effect: $F_{1,52}=22.069, p=0.000$; treatment effect: $F_{1,52}=3.339, p=0.073$; genotype $X$ treatment interaction: $F_{1,52}=9.449, p=0.003$ ) (Fig. 3a3 and 3a3'; genotype effect: $F_{1}$, ${ }_{52}=25.098, p=0.000$; treatment effect: $F_{1,52}=0.286, p=0.595$; genotype $X$ treatment interaction: $\left.F_{1,52}=9.113, p=0.004\right)$. Carbamazepine normalized whole arena locomotion in Fmr1 KO mice to the level observed in WT mice (Fig. 3a1, $p=0.089$ between the treated Fmr1 KO and WT mice). Carbamazepine also normalized hyper-locomotion in the center area of the open field arena in Fmr1 KO mice (Fig. 3a2; $p=0.246$ between the treated Fmr1 KO and WT mice) (Fig. 3a3, $p=0.156$ between the treated Fmr1 KO and WT mice).

In the 3-chamber social interaction test, all groups spent similar total time in the chamber that holds the stranger mouse (Supplementary Fig. 1a) (Fig. 3b1; genotype effect: $F_{1,30}=1.188, p=0.284$; treatment effect: $F_{1,30}=0.002, p=0.968$; genotype $X$ treatment interaction: $\left.F_{1,30}=2.861, p=0.101\right)$. Notably, $F m r 1 \mathrm{KO}$ mice spent less time in direct interaction with the stranger mouse than WT mice (Supplementary Fig. 1b, Fig. 3b2). While a single acute treatment with carbamazepine had no significant effect (Supplementary Fig. 1), repeated daily administration for 8 days improved social interaction in both WT and Fmr1 KO mice. The carbamazepine-treated WT and Fmr1 KO mice showed comparable social interaction (Fig. 3b2; genotype effect: $F_{1,30}=19.521$, $p=0.000$; treatment effect: $F_{1,30}=22.184, p=0.000$; genotype $X$ treatment interaction: $F_{1}$, $30=2.620, p=0.116)$.

Our previous studies found that Fmr1 KO mice made more transitional moves between two chambers in the light/dark test, reflecting hyperactivity and repetitive behavior ${ }^{10,19}$. For this specific behavioral abnormality, neither single (Supplementary Fig. 2a1) nor repeated treatment (Supplementary Fig. 2b1) with carbamazepine exerted significant therapeutic effect. Interestingly, repeated carbamazepine increased total time spent in the light chamber in both WT and Fmr1 KO mice (Supplementary Fig. 2b2). 
Carbamazepine dampens the elevated protein synthesis in Frm1 KO neurons Consistent with previous reports, we found that protein synthesis is elevated in Fmr1 KO hippocampal neurons (Fig. 4a; genotype effect: $F_{1,16}=12.898, p=0.002$ ). Carbamazepine dampened protein synthesis in Fmr1 KO neurons in a dose-dependent manner without affecting wild type neurons (Fig. $4 a$; treatment effect: $F_{3,16}=3.757, p=0.032$; genotype $X$ treatment interaction: $\left.F_{3,16}=5.081, p=0.012\right)$.

Carbamazepine suppresses both ERK1/2 and Akt activity in neurons The level of total ERK1/2 (Fig. $4 \mathrm{~b} 1$ and 4b2; genotype effect: $F_{1,16}=0.027, p=0.871$; treatment effect: $F_{3,16}=0.760, p=0.533$; genotype $X$ treatment interaction: $F_{3,16}=0.303$, $p=0.308$ ) and Akt (Fig. 4c1 and 4c2; genotype effect: $F_{1,16}=0.000, p=0.999$; treatment effect: $F_{3,16}=0.089, p=0.965$; genotype $X$ treatment interaction: $F_{3,16}=0.293, p=0.830$ ) was not affected by genotype and carbamazepine treatment.

As reflected by the levels of phosphorylated ERK1/2 and Akt (i.e. pERK1/2 and pAkt, respectively), the activity of ERK1/2 (Fig. $4 \mathrm{~b} 1$ and $4 \mathrm{~b} 3$; genotype effect: $F_{1,16}=8.788$, $p=0.009$ ) and Akt (Fig. 4c1, and 4c3; genotype effect: $F_{1,16}=57.920, p=0.000$ ) are elevated in Fmr1 KO hippocampal neurons.

Carbamazepine dose-dependently decreases the level of pERK1/2 (Fig. 4b1 and 4b3; treatment effect: $F_{3,16}=129.537, p=0.000$; genotype $X$ treatment interaction: $F_{3,16}=21.401$, $p=0.000$ ) and pAkt (Fig. 4c1 and 4c3; treatment effect: $F_{3,16}=14.840, p=0.000$; genotype $X$ treatment interaction: $\left.F_{3,16}=3.233, p=0.050\right)$ in both WT and $F m r 1 \mathrm{KO}$ neurons. The abnormally elevated pERK1/2 and pAkt in Fmr1 KO neurons were more sensitive to carbamazepine treatment than wild type neurons. Comparing to wild type neurons, pERK1/2 and pAkt in FXS neurons were effectively suppressed by lower concentrations of carbamazepine. While $10 \mu \mathrm{M}$ carbamazepine was sufficient to dampen $\mathrm{pERK} 1 / 2$ and pAkt in Fmr1 KO neuron, higher concentration $(100 \mu \mathrm{M})$ was required for WT neurons (Fig. 4b and 4c).

Pharmacological inhibition of PDE2 and activation of ADCY do not correct the elevated protein synthesis in FXS mouse neurons.

ADCY and PDE have counteracting enzymatic action on CAMP metabolism and may have different effects on cAMP-regulated signaling molecule such as ERK1/2 ${ }^{11,30}$. With regard to the consideration of targeting ADCY1-ERK1/2 for potential therapeutic strategy, one complication is that PDE2A was also identified as an FMRP target ${ }^{16}$. We found that PDE2A is also primarily expressed in neurons but not glial cells (Fig. 5a). Interestingly, PDE2A protein is not altered in adult Fmr1 KO hippocampus (Fig. 5b). This is consistent with that PDE2A may be affected only in juvenile but not adult FXS mice ${ }^{16,31}$.

In contrast to the effects of carbamazepine, BAY607550, which has inhibitory activity against PDE2 ${ }^{32}$, did not show significant effect on protein translation (Fig. 5c: genotype effect: $F_{1,28}=49.538, p=0.000$; treatment effect: $F_{3,28}=1.305, p=0.292$; genotype $X$ treatment interaction: $\left.F_{3,28}=1.770, p=0.176\right)$. As an alternative approach to increase cAMP, forskolin was used to stimulate multiple ADCY. Interestingly, forskolin increased protein translation in both WT and Fmr1 KO neurons (Fig. 5d; genotype effect: $F_{1}$, ${ }_{14}=8.788, p=0.009$; treatment effect: $F_{2,14}=13.539, p=0.001$; genotype $X$ treatment interaction: $F_{2,14}=0.660, p=0.532$ ). 
Effects of PDE2 inhibition and ADCY activation on ERK1/2 and Akt signaling Although the function of ADCY1 in regulating ERK1/2 and Akt signaling has been implicated ${ }^{10}$, the overall effect of cAMP through inhibition of PDE2 or non-specific activation of multiple ADCY subtypes has not been investigated. Consistent with that cAMP positively stimulates pERK1/2, we found that, although there is no effect on total ERK1/2 (Supplementary Fig. 3a1), the PDE2 inhibitor BAY607550 upregulated pERK1/2 in both WT and Fmr1 KO neurons (Fig. 5e1 and 5e2; WT: $F_{3,2}=97.854, p=0.000$; Fmr1 $\left.\mathrm{KO}: F_{3,28}=33.944, p=0.000\right)$. Interestingly, BAY607550 caused decrease in pAkt in both WT and Fmr1 KO neurons (Fig. 5e1 and 5e3; WT: $F_{3,28}=4.072$, $p=0.016$; Fmr1 KO: $F_{3}$, ${ }_{28}=4.970, p=0.007$ ) without affecting the level of total Akt (Supplementary Fig. 3a2).

We found that forskolin causes robust increase of pERK1/2 (Fig. $5 f 1$ and 5f2; WT: $F_{2}$, ${ }_{16}=36.081, p=0.000$; Fmr1 KO: $\left.F_{2,16}=16.800, p=0.000\right)$ but decreased pAkt (Fig. $5 f 1$ and 5f3; WT: $F_{2,16}=11.182, p=0.001$; $F m r 1 \mathrm{KO}: F_{2,16}=20.850, p=0.000$ ) in both WT and $F m r 1$ $\mathrm{KO}$ neurons without affecting total ERK1/2 (Supplementary Fig. 3b1) and total Akt level (Supplementary Fig. 3b2). Together, these results demonstrate that decreasing PDE2A activity or increasing ADCY activity dampens the elevated pAkt but exacerbates the elevated $\mathrm{pEK} 1 / 2$ in FXS mouse neurons.

\section{DISCUSSION}

Our data show that the alteration of cAMP enzymes (i.e. ADCY and PDE) in FXS depends on brain region and developmental stage. Treatment with carbamazepine, which has inhibitory action against ADCY1 is sufficient to correct certain aspects of FXS-associated pathology in a mouse model during adulthood.

As carbamazepine is a pharmacological reagent, it is challenging to definitively pinpoint the mechanism of action underlying the therapeutic effects. Nevertheless, our data show that carbamazepine suppresses both ERK1/2 and Akt activity. This outcome recapitulates the effect of genetic reduction of ADCY1 on rescuing the elevated ERK1/2 and Akt signaling in FXS mouse ${ }^{10}$. It is also consistent with that carbamazepine show pharmacological inhibition activity against ADCY1 ${ }^{15}$. It is important to note that carbamazepine also inhibit ADCY5 and ADCY7 ${ }^{15}$. ADCY7 is not expressed in brain; ADCY5 is predominately expressed in striatum and nucleus accumbens ${ }^{33}$. Functionally, ADCY5 deficiency leads to increase in ERK1/2 activity and mGluR5 signaling ${ }^{33}$, both of which are associated with cellular pathology in FXS ${ }^{5,6,34}$. ADCY 5 deficiency also reduces social interaction ${ }^{33}$. We expect that the carbamazepine effect on ERK/12 and Akt activity in hippocampal neurons and its effect on correcting the social interaction deficits in Fmr1 $\mathrm{KO}$ mice are unlikely mediated through ADCY5 or ADCY7 inhibition.

Along with previous studies, our data show that, while ERK1/2 activity is positively correlated with cAMP level, Akt activity may be specifically regulated by ADCY1 rather than general cAMP level in neurons. It is evident that specific over-expression of ADCY1

${ }^{11}$ or general increase of cAMP by forskolin (Fig. 5f) and PDE2 inhibitors (Fig. 5e) can both increase ERK1/2 activity. However, although genetic reduction of ADCY1 ${ }^{10}$ or carbamazepine treatment decreases Akt activity in FXS samples, general increase of cAMP did not cause increase of Akt activity. Forskolin or PDE2 inhibitor rather decreases Akt activity. Thus, targeting ADCY1 may offer a unique opportunity to concurrently suppress the abnormal ERK1/2 and Akt signaling in FXS. 
It is interesting to note that, although ADCY1, ERK1/2, and Akt are pro-learning molecules and their activity is required for memory formation ${ }^{26,35-37}$, the elevated activity of these signaling molecules is concurrent with the learning deficits in FXS. It is evident that decrease rather than increase of the activity of ADCY1, ERK1/2 and Akt shows therapeutic efficacy to correct cognitive impairments in FXS mouse ${ }^{38,39}$. Although the mechanism remains elusive, it is possible that concurrent increase of ADCY1, ERK1/2 and Akt activity along with other pathological changes in FXS impair rather than facilitate learning.

Treatment strategy through increase of cAMP signaling has been previously proposed 40 , although the level of cAMP in crude homogenates has been found to be normal in both human and mouse FXS samples ${ }^{10,41,42}$. As cAMP homoeostasis is maintained by counteracting enzymatic activities from multiple subtypes of ADCY and PDE, identification of alterations in specific cAMP enzyme may help to better understand the disease mechanism. Further, whether alteration of ADCY and PDE in specific brain region, sub-cellular domain and developmental stage should also be considered. FMRP binds Adcy1 and Pde2a mRNA and the protein levels of ADCY1 and PDE2A are elevated in Fmr1 KO mice ${ }^{8-10,16}$. One complication is that, while elevated ADCY1 is detected in adult FXS mouse samples (Fig. 1), alteration of PDE2A is detected in juvenile samples (i.e. at postnatal day 13) ${ }^{16}$. Notably, ADCY1 is enriched at postsynaptic density ${ }^{43}$ and PDE2A is substantially expressed at the presynaptic active zone ${ }^{16}$. This indicates an age-dependent and compartmentalized alteration of cAMP enzymes in FXS, suggesting tailored therapeutic approaches. Interestingly, it is found that treatment with PDE2 inhibitor BAY607550 rescues social deficits in infant (postnatal day 10-14) and adolescent (postnatal day 30) Fmr1 KO animals ${ }^{32}$. Although PDE4D expression has not been compared between normal and FXS samples, chronic treatment with PDE4D negative allosteric modulator (NAM) BPN14770 improves behavioral symptoms associated with social interaction and perseveration ${ }^{44}$. The effect of PDE2 and PDE4 inhibition on cognitive function such as learning and memory has not been determined. In regards to how inhibition of PDE affects the known signaling alteration such as ERK/12 and Akt, our data show that targeting PDE2 dampens the elevated Akt activity but further increases the elevated ERK1/2 activity in FXS. Moreover, it is worth mentioning that, while ADCYs contribute to cAMP production, PDE2A degrades both CAMP and cGMP. Examination of the therapeutic effect of cGMP may be considered for future investigation.

In summary, our study revealed the efficacy of carbamazepine on correcting the key cellular disease mechanisms including the elevated protein synthesis and ERK1/2 and Akt signaling. Furthermore, we demonstrated therapeutic efficacy of carbamazepine to correct adult behavioral abnormalities in a mouse model of FXS. As carbamazepine is an FDA-approved drug, it may offer a practical approach and be clinically repurposed for FXS therapy. It is worth noting that carbamazepine has been mainly used to treat seizure and neuropathic pain. The increased risk of seizure is observed in about $18 \%$ of the child FXS patients (from as early as 4 months to 9 years of age) but not in adulthood ${ }^{45,46}$. As a symptom- rather than mechanism-based treatment, anticonvulsants including carbamazepine, valproic acid, lamotrigine, and oxcabazepine have been used to control seizure in FXS children without severe side effects ${ }^{47}$. Nonetheless, our results advocate future tests of clinical efficacy with adult human FXS patients, beyond the anticonvalsant effects in children. 


\section{FUNDING AND DISCLOSURE}

This work was supported by R01MH119149 (to HW) from the NIH and W81XWH-17-10270 (to HW and YF) from the Department of Defense. The authors declare no conflict of interest.

\section{AUTHOR CONTRIBUTIONS}

$H W, Q D$, and YF contribute to the conception and design of the study. QD and FZ performed the experiments and analyzed the data. HW, QD, and YF wrote the manuscript.

\section{ACKNOWLEDGEMENTS}

We thank Dr. Zhuang Miao for setting up the object location memory paradigm.

\section{ADDITIONAL INFORMATION}

Supplementary Information accompanies

\section{REFERENCES}

1. Santoro, M.R., Bray, S.M. \& Warren, S.T. Molecular mechanisms of fragile $X$ syndrome: a twenty-year perspective. Annu Rev Pathol 7, 219-245 (2012).

2. Sethna, F., Moon, C. \& Wang, H. From FMRP function to potential therapies for fragile X syndrome. Neurochem Res 39, 1016-1031 (2014).

3. Darnell, J.C. \& Klann, E. The translation of translational control by FMRP: therapeutic targets for FXS. Nat Neurosci (2013).

4. Gross, C., Yao, X., Pong, D.L., Jeromin, A. \& Bassell, G.J. Fragile X mental retardation protein regulates protein expression and mRNA translation of the potassium channel Kv4.2. J Neurosci 31, 5693-5698 (2011).

5. Wang, X., et al. Activation of the extracellular signal-regulated kinase pathway contributes to the behavioral deficit of fragile x-syndrome. J Neurochem 121, 672679 (2012).

6. Michalon, A., et al. Chronic pharmacological mGlu5 inhibition corrects fragile $\mathrm{X}$ in adult mice. Neuron 74, 49-56 (2012).

7. Pellerin, D., et al. Lovastatin corrects ERK pathway hyperactivation in fragile $\mathrm{X}$ syndrome: potential of platelet's signaling cascades as new outcome measures in clinical trials. Biomarkers 21, 497-508 (2016).

8. Ascano, M., Jr., et al. FMRP targets distinct mRNA sequence elements to regulate protein expression. Nature 492, 382-386 (2012).

9. Darnell, J.C., et al. FMRP stalls ribosomal translocation on mRNAs linked to synaptic function and autism. Cell 146, 247-261 (2011).

10. Sethna, F., et al. Enhanced expression of ADCY1 underlies aberrant neuronal signalling and behaviour in a syndromic autism model. Nat Commun 8, 14359 (2017). 
11. Wang, H., Ferguson, G.D., Pineda, V.V., Cundiff, P.E. \& Storm, D.R. Overexpression of type-1 adenylyl cyclase in mouse forebrain enhances recognition memory and LTP. Nat Neurosci 7, 635-642 (2004).

12. Chen, X., Cao, H., Saraf, A., Zweifel, L.S. \& Storm, D.R. Overexpression of the type 1 adenylyl cyclase in the forebrain leads to deficits of behavioral inhibition. $J$ Neurosci 35, 339-351 (2015).

13. Gross, C. \& Bassell, G.J. Excess protein synthesis in FXS patient lymphoblastoid cells can be rescued with a p110beta-selective inhibitor. Molecular medicine 18, 336-345 (2012).

14. Gross, C., et al. Excess phosphoinositide 3-kinase subunit synthesis and activity as a novel therapeutic target in fragile X syndrome. J Neurosci 30, 10624-10638 (2010).

15. Mann, L., et al. Inhibition of specific adenylyl cyclase isoforms by lithium and carbamazepine, but not valproate, may be related to their antidepressant effect. Bipolar Disord 11, 885-896 (2009).

16. Maurin, T., et al. HITS-CLIP in various brain areas reveals new targets and new modalities of RNA binding by fragile $X$ mental retardation protein. Nucleic Acids Res 46, 6344-6355 (2018).

17. Zhou, X., Lin, D.S., Zheng, F., Sutton, M.A. \& Wang, H. Intracellular calcium and calmodulin link brain-derived neurotrophic factor to p70S6 kinase phosphorylation and dendritic protein synthesis. J Neurosci Res 88, 1420-1432 (2010).

18. Chen, S.H., Oyarzabal, E.A. \& Hong, J.S. Preparation of rodent primary cultures for neuron-glia, mixed glia, enriched microglia, and reconstituted cultures with microglia. Methods Mol Biol 1041, 231-240 (2013).

19. Ding, Q., Sethna, F. \& Wang, H. Behavioral analysis of male and female Fmr1 knockout mice on C57BL/6 background. Behav Brain Res 271, $72-78$ (2014).

20. Vogel-Ciernia, A. \& Wood, M.A. Examining object location and object recognition memory in mice. Curr Protoc Neurosci 69, 831 31-17 (2014).

21. Moy, S.S., et al. Sociability and preference for social novelty in five inbred strains: an approach to assess autistic-like behavior in mice. Genes Brain Behav 3, 287302 (2004).

22. Gantois, I., et al. Metformin ameliorates core deficits in a mouse model of fragile $X$ syndrome. Nat Med 23, 674-677 (2017).

23. Yang, Y.M., et al. Identification of a molecular locus for normalizing dysregulated GABA release from interneurons in the Fragile X brain. Mol Psychiatry (2018).

24. Schmidt, E.K., Clavarino, G., Ceppi, M. \& Pierre, P. SUnSET, a nonradioactive method to monitor protein synthesis. Nat Methods 6, 275-277 (2009).

25. Xia, Z., Choi, E.J., Wang, F., Blazynski, C. \& Storm, D.R. Type I calmodulinsensitive adenylyl cyclase is neural specific. J Neurochem 60, 305-311 (1993).

26. Wang, H. \& Zhang, M. The role of $\mathrm{Ca}(2)(+)$-stimulated adenylyl cyclases in bidirectional synaptic plasticity and brain function. Rev Neurosci 23, 67-78 (2012).

27. Qin, M., Kang, J. \& Smith, C.B. A null mutation for Fmr1 in female mice: effects on regional cerebral metabolic rate for glucose and relationship to behavior. Neuroscience 135, 999-1009 (2005). 
28. Assini, F.L., Duzzioni, M. \& Takahashi, R.N. Object location memory in mice: pharmacological validation and further evidence of hippocampal CA1 participation. Behav Brain Res 204, 206-211 (2009).

29. Barrett, R.M., et al. Hippocampal focal knockout of CBP affects specific histone modifications, long-term potentiation, and long-term memory. Neuropsychopharmacology : official publication of the American College of Neuropsychopharmacology 36, 1545-1556 (2011).

30. Zhang, H.T., et al. Inhibition of the phosphodiesterase 4 (PDE4) enzyme reverses memory deficits produced by infusion of the MEK inhibitor U0126 into the CA1 subregion of the rat hippocampus. Neuropsychopharmacology : official publication of the American College of Neuropsychopharmacology 29, 1432-1439 (2004).

31. Tang, B., et al. Fmr1 deficiency promotes age-dependent alterations in the cortical synaptic proteome. Proc Natl Acad Sci U S A 112, E4697-4706 (2015).

32. Maurin, T., et al. Involvement of Phosphodiesterase 2A Activity in the Pathophysiology of Fragile X Syndrome. Cereb Cortex (2018).

33. Kim, H., et al. Loss of Adenylyl Cyclase Type-5 in the Dorsal Striatum Produces Autistic-Like Behaviors. Mol Neurobiol 54, 7994-8008 (2017).

34. Bear, M.F., Huber, K.M. \& Warren, S.T. The mGluR theory of fragile X mental retardation. Trends Neurosci 27, 370-377 (2004).

35. Sweatt, J.D. Mitogen-activated protein kinases in synaptic plasticity and memory. Curr Opin Neurobiol 14, 311-317 (2004).

36. Sui, L., Wang, J. \& Li, B.M. Role of the phosphoinositide 3-kinase-Akt-mammalian target of the rapamycin signaling pathway in long-term potentiation and trace fear conditioning memory in rat medial prefrontal cortex. Learn Mem 15, 762-776 (2008).

37. Chen, X., et al. PI3 kinase signaling is required for retrieval and extinction of contextual memory. Nat Neurosci 8, 925-931 (2005).

38. Gross, C., et al. Selective role of the catalytic PI3K subunit p110beta in impaired higher order cognition in fragile X syndrome. Cell Rep 11, 681-688 (2015).

39. Asiminas, A., et al. Sustained correction of associative learning deficits after brief, early treatment in a rat model of Fragile X Syndrome. Sci Transl Med 11(2019).

40. Kelley, D.J., et al. The cyclic AMP phenotype of fragile $X$ and autism. Neuroscience and biobehavioral reviews 32, 1533-1543 (2008).

41. Kelley, D.J., et al. The cyclic AMP cascade is altered in the fragile $X$ nervous system. PLoS One 2, e931 (2007).

42. Berry-Kravis, E. \& Sklena, P. Demonstration of abnormal cyclic AMP production in platelets from patients with fragile X syndrome. Am J Med Genet 45, 81-87 (1993).

43. Conti, A.C., et al. Distinct regional and subcellular localization of adenylyl cyclases type 1 and 8 in mouse brain. Neuroscience 146, 713-729 (2007).

44. Gurney, M.E., Cogram, P., Deacon, R.M., Rex, C. \& Tranfaglia, M. Multiple Behavior Phenotypes of the Fragile-X Syndrome Mouse Model Respond to Chronic Inhibition of Phosphodiesterase-4D (PDE4D). Sci Rep 7, 14653 (2017).

45. Musumeci, S.A., et al. Epilepsy and EEG findings in males with fragile $X$ syndrome. Epilepsia 40, 1092-1099 (1999).

46. Berry-Kravis, E. Epilepsy in fragile X syndrome. Dev Med Child Neurol 44, 724728 (2002). 
47. Hagerman, R.J., et al. Advances in the treatment of fragile $X$ syndrome. Pediatrics 123, 378-390 (2009).

\section{FIGURE LEGENDS}

Fig. 1, Ding et al.
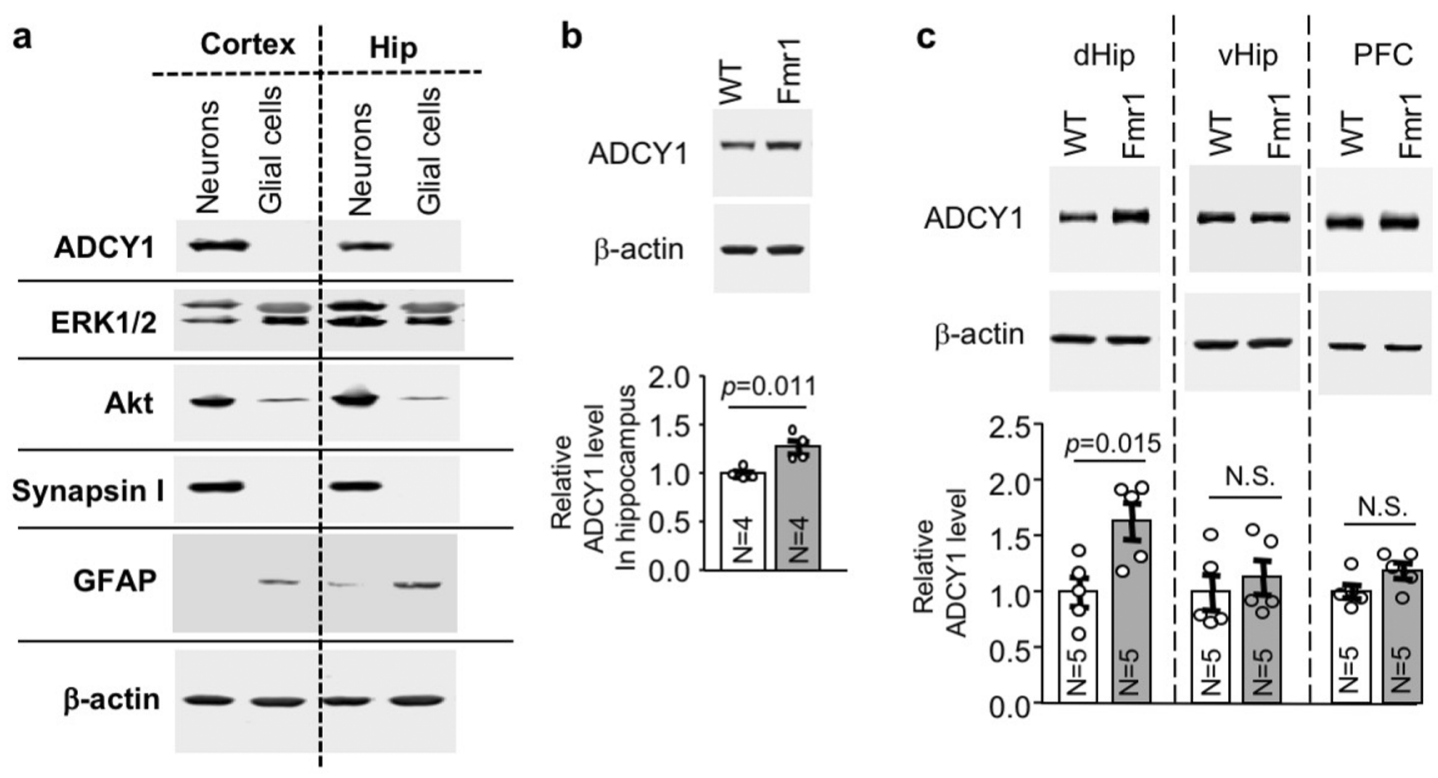

Fig. 1 ADCY1 expression in wild type and Fmr1 knockout samples. a. ADCY1 is expressed in neurons but not in glial cells. Primary cultures of neuron or glial cell were obtained from new born cortex and hippocampus. Cells were harvested on DIV (days in vitro) 14. Expression of ADCY1, ERK1/2, Akt, synapsin I (a neuronal marker protein), GFAP (a glial cell marker protein), and $\beta$-actin in neuronal or glial preparations were determined by Western blot analysis. b. ADCY1 protein expression is elevated in the hippocampus of adult $F m r 1 \mathrm{KO}$ mice. Hippocampal tissues were collected from 3-month old wild type (WT) and Fmr1 KO (Fmr1) mice. The expression of ADCY1 was determined by Western blot and normalized to the level of $\beta$-actin. c. ADCY1 expression in the dorsal hippocampus (dHip), ventral hippocampus (vHip), and prefrontal cortex (PFC) of 3-month WT and Fmr1 KO mice. The level of ADCY1 was normalized with $\beta$-actin. Data are presented as average +/- SEM. The $p$ values were determined by Student's t-test. N.S.: not significant. 
Fig. 2, Ding et al.
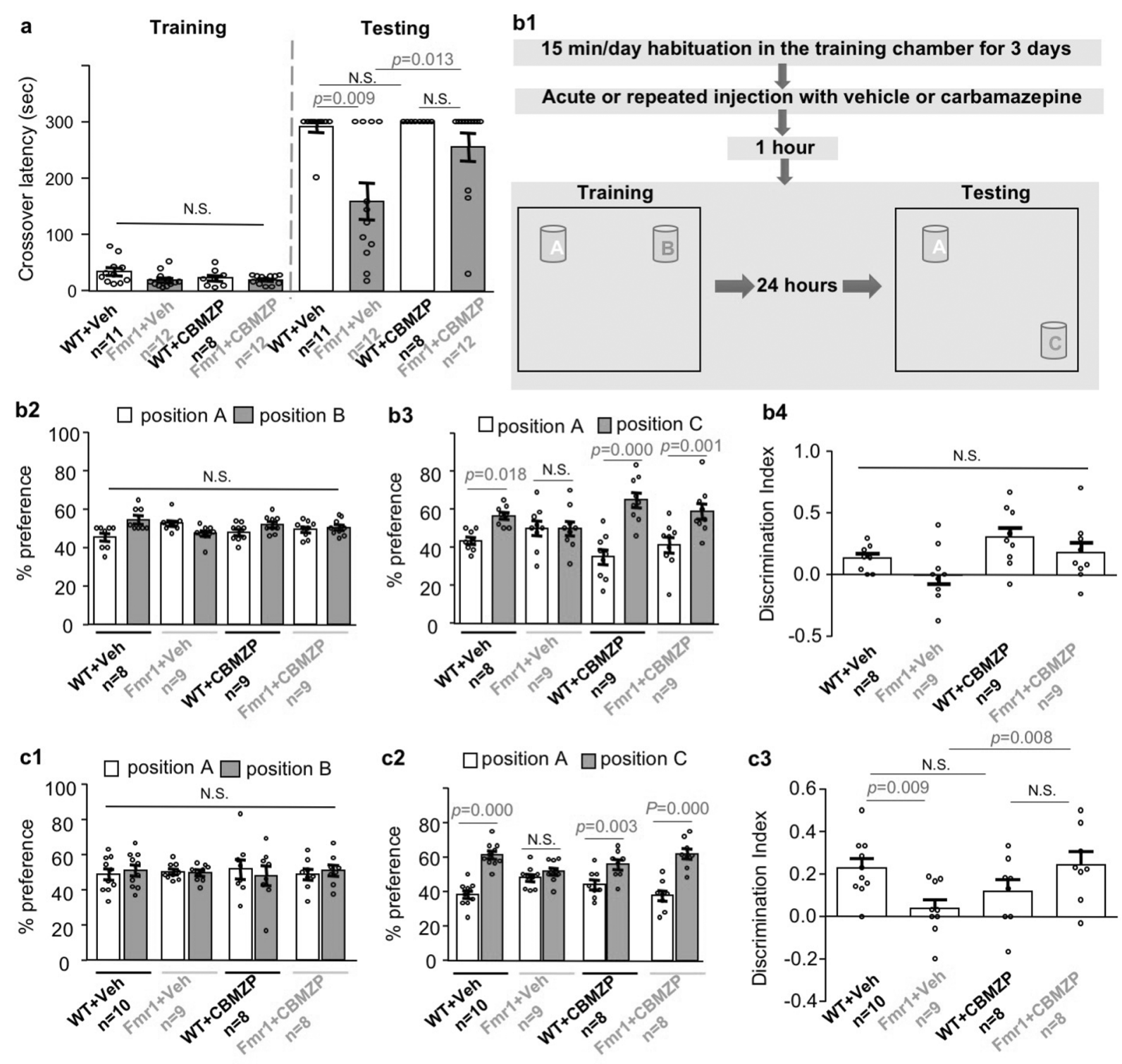

Fig. 2 Carbamazepine restores passive avoidance and object location memory in FXS mice. a. Carbamazepine improves passive avoidance memory in FXS mice. Wild type (WT) and Fmr1 KO (Fmr1) mice were first injected with vehicle (Veh) or carbamazepine (CBMZP). One hour later, they were training by the passive avoidance paradigm and then returned to their home cage. Twenty-four hours later, they were tested. Crossover latency was recorded during training and testing. $\mathbf{b}$ and $\mathbf{c}$. Carbamazepine improves object location memory in FXS mice. Treatment and behavioral paradigm is shown in b1. Mice were trained (b2 or c1) and tested (b3 and b4 or c2 and c3) for object location memory after receiving a single (b2 to b4) or repeated (c1 to c3) injection with vehicle or carbamazepine. Their preference to the objects during training (b2 and $\mathbf{c 1}$ ) and testing (b3 and c2) is shown. The object discrimination index during testing is shown in b4 and 
c3. All data are presented as average +/- SEM. Two-way ANOVA followed by post hoc analysis is use for the passive avoidance training data (a) and the object discrimination index data (b4 and $\mathbf{c} 3$ ). Fisher exact is used for data collected during passive avoidance testing. Three-way ANOVA followed by post hoc analysis is used to analyze the object location training/testing data. N.S.: not significant. 
Fig. 3, Ding et al.
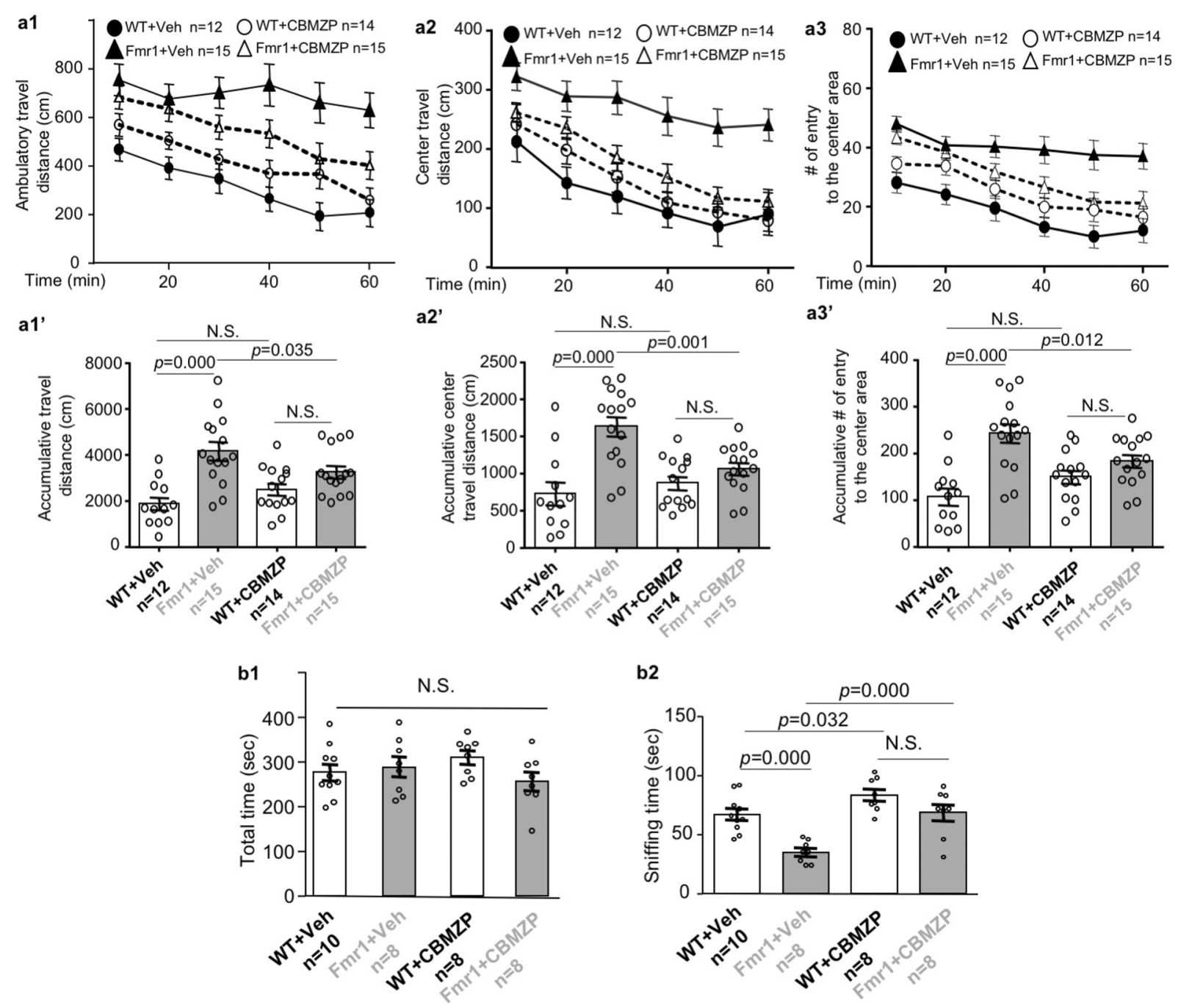

Fig. 3 Carbamazepine corrects hyper locomotion and social deficits in FXS mice. Wild type (WT) and Fmr1 KO mice received a single (a) or repeated (once/day for 8 days) (b) injection with vehicle (Veh) or carbamazepine (CBMZP). a. 60 min after the injection, mice were subjected to a 1-hour open filed test. The ambulatory travel distance in the whole open field arena and center area for each of the 10-min bin is presented in a1 and a2, respectively. Number of entry to the center area for each of the 10-min bin is presented in a3. The corresponding cumulative activity during the whole 1-hour testing is presented in a1', a2', and a3'. b. 60 min after the last daily injection, mice were subjected to a 3chamber social interaction test. Total time spent in the stimulus mouse chamber is presented in b1. Direct social interaction between the test mouse and the stimulus mouse was determined by the amount of sniffing time and presented in b2. Data are presented as average +/- SEM. Three-way (a1, a2, and a3) or two-way ANOVA (a1', a2', a3', b1, and b2) were used for data analysis. Difference between two groups was determined by post hoc analysis following ANOVA test. N.S.: not significant. 
Fig. 4, Ding et al.

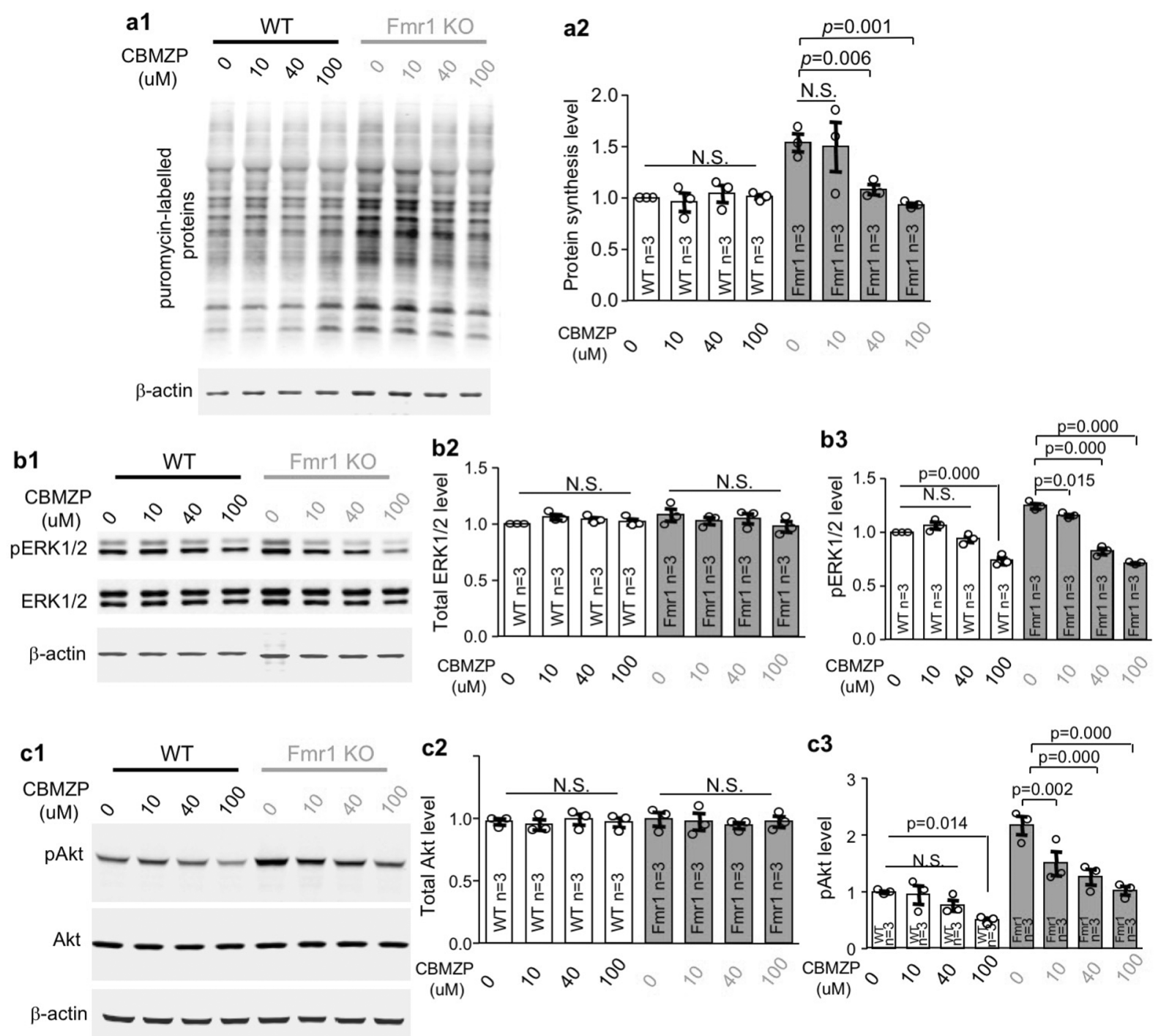

Fig 4. Carbamazepine corrects the aberrantly elevated protein synthesis and neuronal signaling in FXS mouse neurons. DIV (days in vitro) 14 neurons cultured from hippocampus of wild type (WT) and Fmr1 KO mice were treated with various concentrations of carbamazepine (CBMZP, as indicated). Samples were harvested 60 min after treatment. a. Protein synthesis was labelled with puromycin and determined by Western blot with antibody against puromycin. The level of puromycin-labelled protein synthesis was normalized to $\beta$-actin. $\mathbf{b}$ and $\mathbf{c}$. The level of total ERK1/2 and pERK1/2 (b) and total Akt and pAkt (c) was determined by Western blot. The level of ERK1/2 and Akt was normalized to $\beta$-actin. The level of $p E R K 1 / 2$ and pAkt was normalized to total ERK1/2 and Akt, respectively. Representative results are presented in $\mathbf{a 1}$, b1, and c1. Quantification is presented as average +/- SEM in a2, b2, b3, c2 and c3. The $p$ values were determined by two-way ANOVA followed by post-hoc analysis. N.S.: not significant. 
Fig. 5, Ding et al.
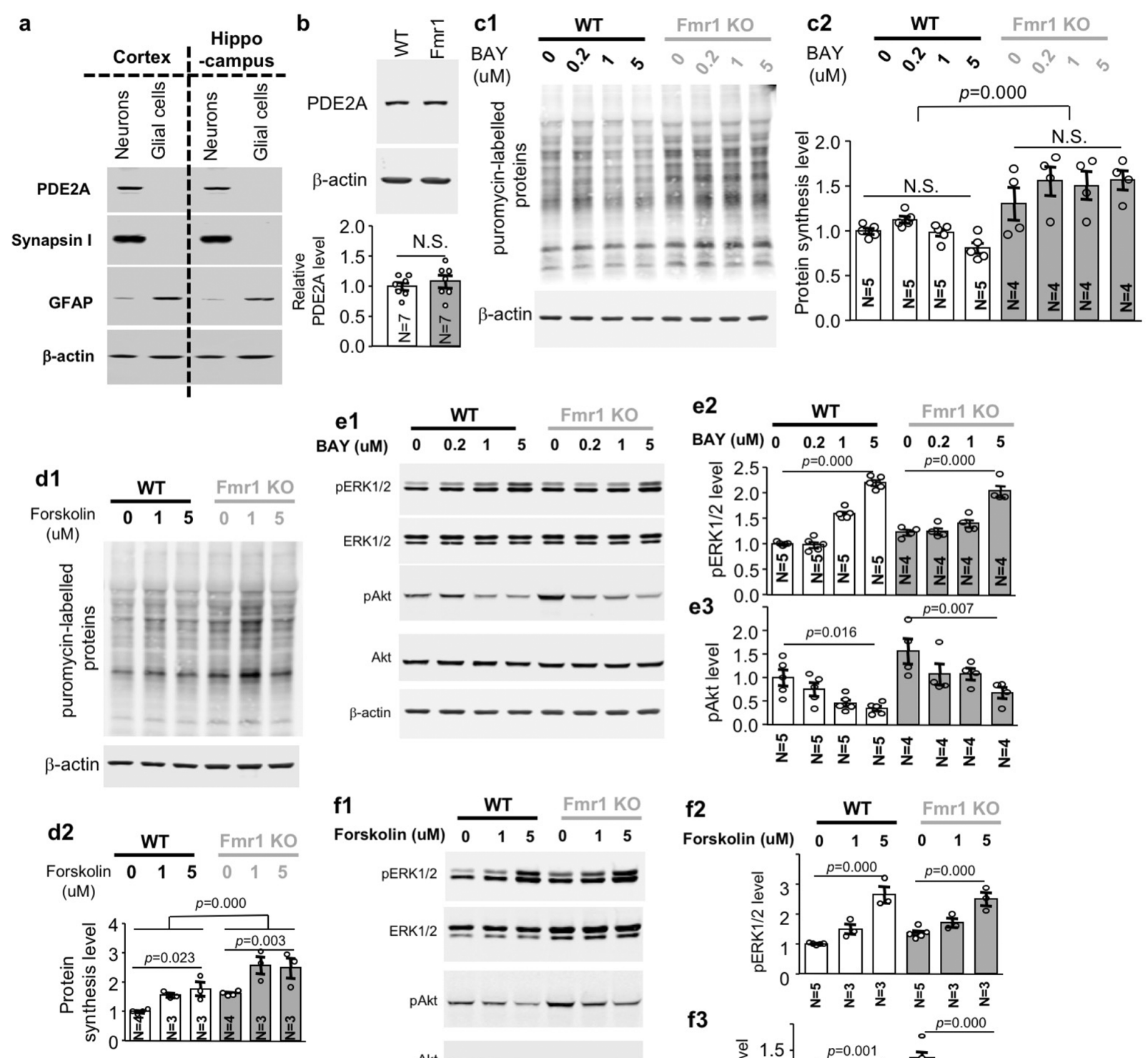

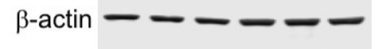
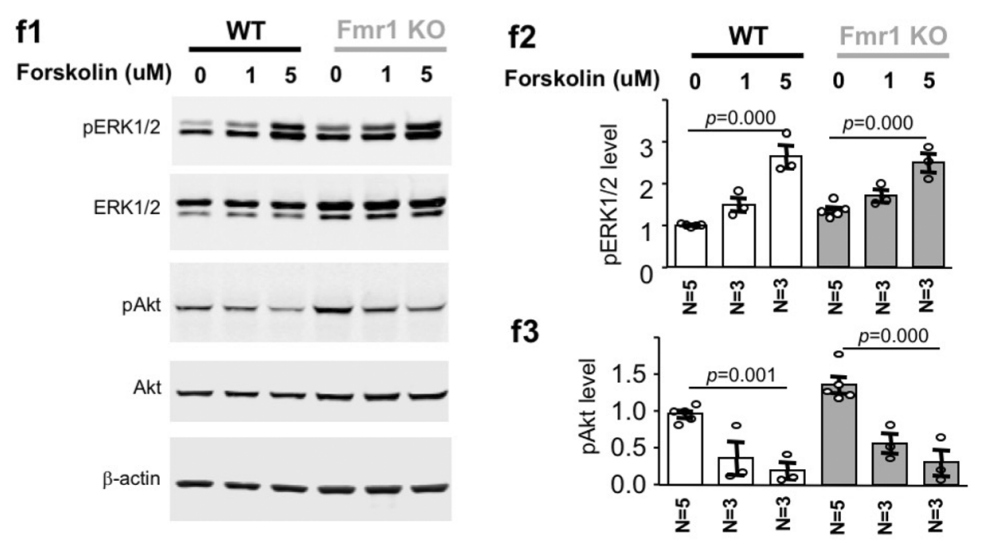

Fig 5. Effects of pharmacological inhibition of PDE2 and general activation of ADCY on protein synthesis and ERK1/2 and Akt activity. a. PDE2A is expressed in neurons but not in glial cells. As described in Fig. 1a, the expression of PDE2A, synapsin I, GFAP, and $\beta$ actin in neuronal or glial preparations were determined by Western blot analysis. $\mathbf{b}$. PDE2A protein is expressed at comparable level in the hippocampus of 3-month old wild type (WT) and Fmr1 KO (Fmr1) mice. The level of PDE2A was normalized with $\beta$-actin. c to $\mathbf{f}$, DIV 14 neurons from WT and Fmr1 KO mice were treated with PDE2 inhibitor BAY607550 (BAY) (c and e), and ADCY stimulator forskolin (d and f) at various concentrations (as indicated) for 1 hour. $\mathbf{c}$ and $\mathbf{d}$. Protein synthesis was labelled with 
puromycin and determined by Western blot with antibody against puromycin. Representative results are shown in $\mathbf{c 1}$ and d1. Quantification (normalized with $\beta$-actin) is shown in $\mathbf{c} 2$ and $\mathbf{d 2}$. e and $\mathbf{f}$. The level of total ERK1/2, pERK1/2, total Akt, pAkt, and $\beta$-actin was determined by Western blot. The level of pERK1/2 and pAkt was normalized to total ERK1/2 and Akt, respectively. Representative results are shown in $\mathbf{e 1}$ and $\mathbf{f 1 .}$ Quantification is presented as average +/- SEM. Quantification for $\mathrm{pERK} 1 / 2$ is presented in e2 and f2. Quantification for pAkt is presented in e3 and f3. The $p$ values were determined by Student's t-test (b), two-way (c to f) ANOVA followed by post-hoc analysis. N.S.: not significant. 


\section{Supplementary Fig. 1, Ding et al.}
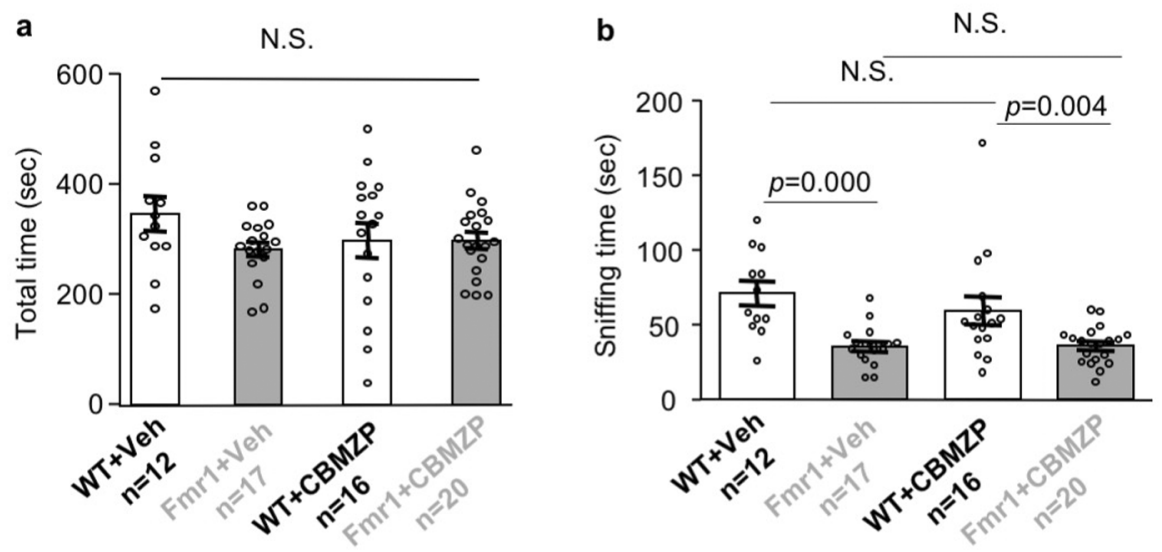

Supplementary Fig. 1 Single carbamazepine administration has no effects on social interaction. Wild type (WT) and Fmr1 KO mice were injected with vehicle (Veh) or carbamazepine (CBMZP). 60 min after the injection, mice were subjected to a 3-chamber social interaction test. Total time spent in the stimulus mouse chamber and direct social interaction are presented in $\mathbf{a}$ and $\mathbf{b}$, respectively. Data are presented as average +/SEM. a. there is no difference in total time among all groups (genotype effect: $F_{1,61}=1.944$, $p=0.168$; treatment effect: $F_{1,61}=0.481, p=0.491$; genotype $X$ treatment interaction: $F_{1}$, $\left.{ }_{61}=1.899, p=0.173\right)$. b. Fmr1 KO mice show social deficits and are not affected by carbamazepine (genotype effect: $F_{1,61}=24.088, p=0.000$; treatment effect: $F_{1,61}=0.864$, $p=0.361$; genotype $X$ treatment interaction: $F_{1,61}=0.946, p=0.335$ ). Difference between two groups was determined by post hoc analysis following two-way ANOVA test. N.S.: not significant. 


\section{Supplementary Fig. 2, Ding et al.}

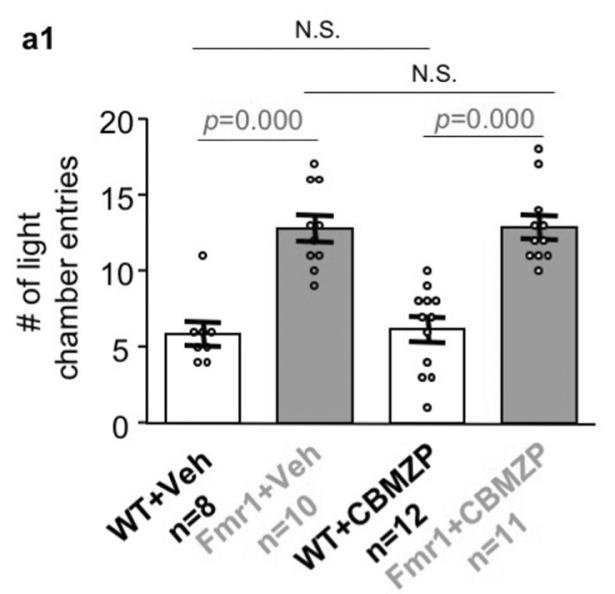

a2
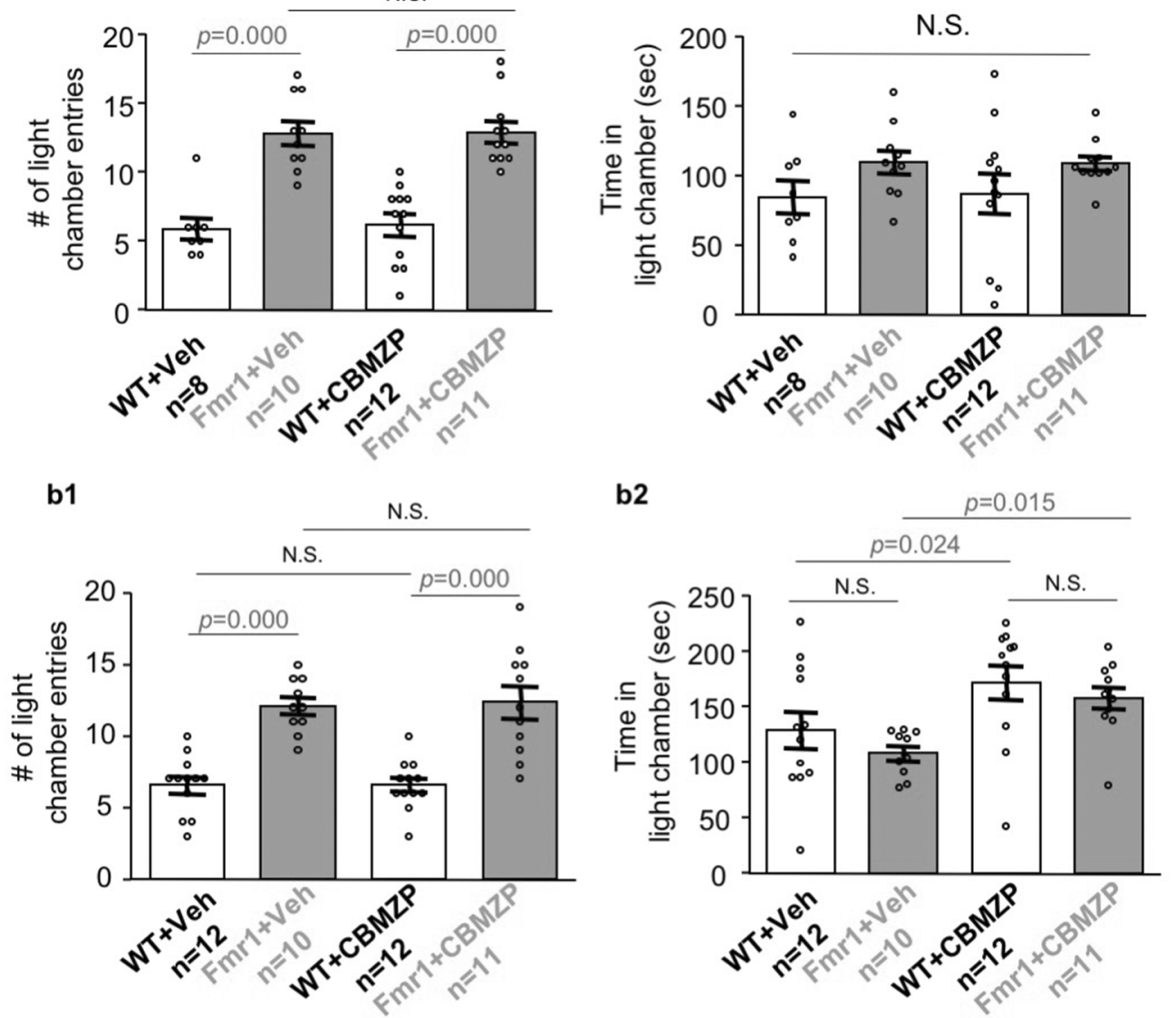

Supplementary Fig. 2 Carbamazepine does not have effect on the abnormal light-dark test behavior in Fmr1 KO mice. Wild type (WT) and Fmr1 KO mice were i.p. injected with vehicle (Veh) or carbamazepine (CBMZP) once (a) or daily for 8 days (b). 60 min after the single injection (a) or the last daily injection (b), mice were subjected to a 5-min light dark test, during which animals were allowed to travel freely between the light and dark chamber. Number of transition made between the light and dark chamber was recorded and presented in a1 (genotype effect: $F_{1,37}=68.412, p=0.000$; drug effect: $F_{1,37}=0.059$, $p=0.81$; genotype $X$ drug interaction: $F_{1,37}=0.012, p=0.913$ ) and b1 (genotype effect: $F_{1}$, ${ }_{41}=57.775, p=0.000$; drug effect: $F_{1,41}=0.031, p=0.860$; genotype $X$ drug interaction: $F_{1}$, ${ }_{41}=0.031, p=0.860$ ). Total time spent in the light chamber is presented in a2 (genotype effect: $F_{1,37}=4.510, p=0.04$; drug effect: $F_{1,37}=0.005, p=0.944$; genotype $X$ drug interaction: $F_{1,37}=0.020, p=0.888$ ) and b2 (genotype effect: $F_{1,41}=1.677, p=0.230$; drug effect: $F_{1,41}=11.874, p=0.001$; genotype $X$ drug interaction: $F_{1,41}=0.063, p=0.804$ ). Data are presented as average +/- SEM. Difference between two groups was determined by post hoc analysis following two-way ANOVA test. N.S.: not significant. 
Supplementary Fig. 3, Ding et al.
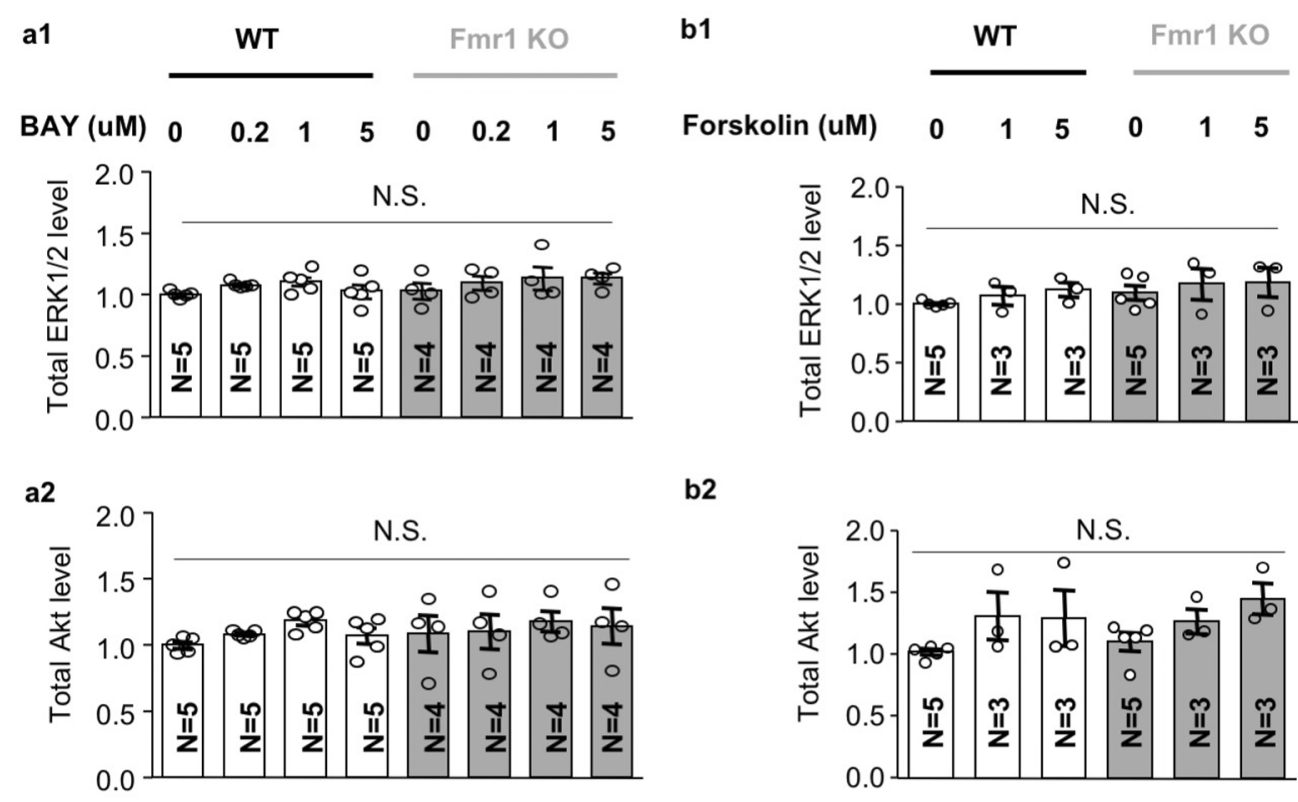

b2
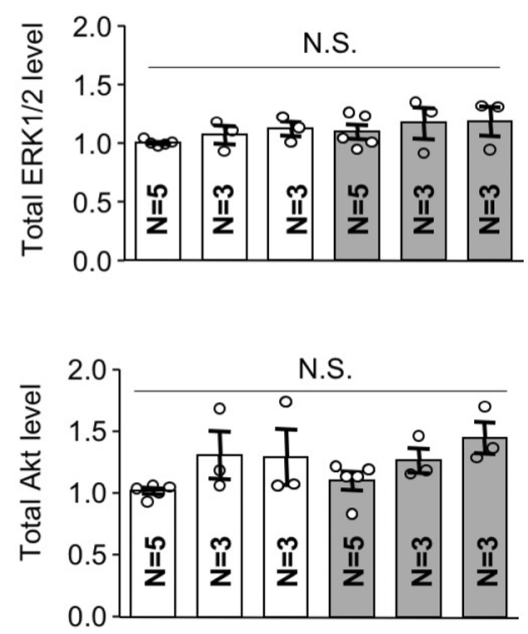

Supplementary Fig. 3 Effects of BAY607550 (a) and forskolin (b) on the level of total ERK1/2 and Akt in wild type and Fmr1 KO neurons. DIV 14 neurons were treated with BAY607550 and forskolin as described in Fig. 5. Quantification of ERK/12 (a1 and b1) and Akt (a2, and b2) is presented as average +/- SEM following normalization with $\beta$ actin. $p$ values were determined by two-way ANOVA. N.S.: not significant. a1. genotype effect: $F_{1,28}=1.639, p=0.211$; drug effect: $F_{3,28}=1.535, p=0.227$; genotype $X$ drug interaction: $F_{3,28}=0.322, p=0.809$. a2. genotype effect: $F_{1,28}=0.630, p=0.434$; drug effect: $F_{3,28}=0.963, p=0.424$; genotype $X$ drug interaction: $F_{3,28}=0.143, p=0.933$. b1. genotype effect: $F_{1,16}=1.949, p=0.182$; drug effect: $F_{2,16}=1.097, p=0.358$; genotype $X$ drug interaction: $F_{2,16}=0.024, p=0.976$. b2. genotype effect: $F_{1,16}=0.473, p=0.501$; drug effect: $F_{2,16}=4.320, p=0.032$; genotype $X$ drug interaction: $F_{2,16}=0.317, p=0.733$. 